Meta

Journal des traducteurs

Translators' Journal

\title{
La traduction : regard linguistique et sémiotique
}

\section{Vjaceslav V. Ivanov}

Volume 37, numéro 1, mars 1992

La traduction en Russie : théorie et pratique / Translation in Russia: Theory and Practice

URI : https://id.erudit.org/iderudit/003233ar

DOI : https://doi.org/10.7202/003233ar

Aller au sommaire du numéro

Éditeur(s)

Les Presses de l'Université de Montréal

ISSN

0026-0452 (imprimé)

1492-1421 (numérique)

Découvrir la revue

Citer cet article

Ivanov, V. V. (1992). La traduction : regard linguistique et sémiotique. Meta, 37(1), 9-17. https://doi.org/10.7202/003233ar d'utilisation que vous pouvez consulter en ligne.

https://apropos.erudit.org/fr/usagers/politique-dutilisation/ 


\title{
LA TRADUCTION : REGARD LINGUISTIQUE ET SÉMIOTIQUE
}

\author{
VJACESLAV V. IVANOV \\ Institut d'études slaves et balkaniques \\ Académie des sciences de l'URSS, Moscou, URSS
}

Le lien qui attache la science moderne du langage à la traduction littéraire, notamment poétique, est double. D'une part, grâce aux acquisitions de la linguistique structurale et mathématique, des modèles sont désormais concevables qui élucident les prémisses langagières de la traduction poétique, ses potentialités et problèmes. D'autre part, s'exerce une action en retour: la traduction poétique peut servir de modèle de situations fréquentes dans la science moderne. Précurseur de la cybernétique, P. Florenski ${ }^{1}$ écrivait dès 1922 dans l'un de ses travaux mathématiques: «Nous le savons, tout comme plusieurs versions d'une cuvre poétique dans une autre langue ne font pas obstacle l'une à l'autre, mais au contraire se complètent l'une l'autre, encore qu'aucune ne remplace tout à fait le texte original, les visions scientifiques d'une seule et même réalité peuvent et doivent être multiples, et cela sans nuire aucunement à la vérité» (1922: 7), idée proche du principe de complémentarité que devait formuler Niels Bohr. Ainsi, le principe de la non-unicité des descriptions phonologiques d'un même système phonétique, découvert par Yuen-Ren Chao (1958), est un important acquis de la linguistique moderne. La pluralité des versions de la poésie orientale classique est elle aussi une tradition démonstrative. Les meilleures des versions russes, que nous devons à V. Alexeiev (1916), comportent des traductions en prose, paraphrases autant que commentaires du texte de départ. C'est aussi à $\mathrm{V}$. Alexeiev que revient l'idée que la traduction interlinguale d'un texte difficile gagne à être précédée d'une traduction intralinguale: mieux vaut, disait-il, traduire les Aphorismes de Confucius en russe non pas directement mais en passant par le truchement du célèbre philosophe Zhu Xi (XII ${ }^{\mathrm{e}}$ siècle), s'en servant comme d'un tremplin pour un saut périlleux ${ }^{2}$. Qu'une version intralinguale (c'est-à-dire la transformation d'un texte en un autre texte qui en rend le contenu) puisse précéder une traduction proprement dite, l'histoire littéraire le prouve. Les cas ne sont pas rares, en effet, d'écrivains variant en prose et en vers un seul et même thème. Ainsi, Pasternak a exprimé le sentiment éprouvé à la première approche de Venise par deux poèmes dont l'un reprend l'autre en le transformant, ainsi que dans un fragment en prose de «Sauf-conduit»; dans l'autobiographie rédigée peu avant sa mort, il se souvient de ces trois essais de récupération. S'attaquant à l'une de ces trois versions, le traducteur devra avoir présentes à l'esprit les deux autres. C'est que le contenu est également porté par chacune d'elles, et ce n'est pas tant par le sens qu'elles se distinguent que par la mise en texte.

La pluralité des versions possibles d'un même texte poétique, aussi bien que la variation d'un même thème par le poète et la paraphrase intralinguale d'un texte complexe, relève d'une propriété essentielle de la langue naturelle, étudiée par la sémantique linguistique moderne: toute langue possède des ressources synonymiques pour exprimer une même signification. Et c'est ce qui distingue la langue naturelle des langages informationnels et logiques scientifiques, qui visent à la monosémie totale. Le coefficient $h_{2}$, qui mesure la diversité des moyens véhiculant le même contenu, peut en 
principe être calculé à partir d'expériences telles que les différentes versions d'un même texte, notamment poétique. Dans ce dernier cas, ce qui importe tout d'abord, c'est le rapport entre le coefficient $h_{2}$ et le coefficient $B$ qui définit les contraintes prosodiques qui limitent le nombre des textes exprimant une même idée par des moyens langagiers différents.

Faisant valoir pour l'étude du vers les méthodes de la théorie de l'information, A. Kolmogorov partait de l'idée (conforme aux formules de Claude Shannon élaborées par d'autres auteurs au cours de ces deux dernières décennies) que l'entropie de la langue naturelle peut être expérimentalement définie ${ }^{3}$. Selon Kolmogorov, l'entropie de la langue $H$ est la somme de $h_{1}$, capacité informationnelle de la langue (c'est-à-dire la quantité de pensées différentes que peut véhiculer un texte de longueur donnée), et $h_{2}$, mesure des ressources dont dispose cette langue pour porter la même signification: $H=h_{1}+h_{2}$. Si le coefficient $B$, qui définit les contraintes formelles imposées par la versification, est supérieur à la mesure synonymique de la langue $\left(B>h_{2}\right)$, la communication des significations ainsi codées (et, partant, une traduction poétique adéquate) est impossible. C'est dire de manière converse que l'inéquation $h_{2}>\beta$ est une condition nécessaire de la création poétique et de la traduction poétique (de ce point de vue, cette dernière ne se distingue pas de l'écriture originale). La même inéquation détermine le rapport entre la complexité de la forme poétique (c'est-à-dire de la grandeur B) et le nombre des emplois figurés des mots qui augmentent la grandeur $h_{2}$. Une augmentation n'est possible que dans la mesure où la structure de la langue permet d'élargir le domaine des emplois imaginaires (figurés ou métaphoriques) du mot sans que se confonde pour autant la signification du mot en question avec celle d'un autre mot du même champ sémantique: lion doit toujours se distinguer de tigre mais peut signifier «héros». Une métaphorisation et une figuration accrues permettent d'augmenter le nombre des contraintes formelles (rimes, assonances, etc.) dans le texte donné. C'est ce qui explique le rapport entre la complexité formelle et l'imagerie propre aux traditions poétiques orientales et à certaines époques dans l'histoire de la poésie européenne. Une poésie à mètre et forme strophique fixes, par exemple la ballade en ancien français, le sonnet classique italien, espagnol et français, impose au poète un langage richement imagé. On comprend d'autre part que le vers libre soit si courant dans la poésie politique et intellectuelle, où les termes ont un sens bien défini, comme par exemple dans les vers de Brecht. La faveur du vers libre dans la poésie moderne est en relation avec l'opposition atténuée entre le langage parlé et l'écrit poétique. Vu sous cet angle, le vers libre du $\mathrm{Xx}^{\mathrm{c}}$ siècle rappelle le vers blanc des siècles précédents (là comme ici, l'affaiblissement des contraintes imposées par l'organisation du vers peut être compensé par une structure sémantique plus complexe du texte). La tendance au rapprochement entre le langage parlé et le langage poétique ne date pas d'hier mais ce sont les grands poètes de notre siècle qui ont nettement pris conscience de cette régularité. Dans ses articles des années 1920 sur la poésie, Mandelstam a révélé la tendance capitale du langage poétique russe, sa «sacralisation», son rapprochement du langage commun, pensée par ailleurs proche de l'interprétation de T. S. Eliot. Ces réflexions devaient préparer le terrain à la victoire du vers libre dans la poésie de la génération future.

Les contraintes à la structure phonique du vers libre et à celle du vers sans rimes ne valent pas pour chaque vers, comme c'est le cas dans la versification classique. Elles le sont d'autant moins pour le traducteur. Traduisant les vers de César Vallejo:

Un hombre pasa con un pan al hombro.

¿Voy a escribar, despues, sobre mi doble? 
Enzenberger omet des assonances de la voyelle $o$ avec les labiales suivantes et $r$ (ou $l$ ) (hombre-hombro-sobre-doble) pour ne rendre que le sens et la moyenne du nombre des syllabes, autant que faire se peut:

Ein Mann geht vorbei mit einem Brot auf der Schulter.

Wie soll ich da über mein Double schreiben ${ }^{4}$ ?

On aura remarqué que ces deux vers allemands sont autrement tournés que les vers espagnols. C'est d'ailleurs de la même façon que procèdent bon nombre de traducteurs français de la poésie russe qui substituent à la structure phonique du vers russe un vers délivré des contraintes prosodiques. Cependant, la version des deux vers suivants de cette même pièce reconstitue exactement le sens autant que la forme (marquée négativement par l'absence de toute contrainte, sinon celle de la longueur moyenne du vers):

Alguien va en un entierre sollozando.

¿Cómo ingresar a la Academia?

Irgend jemand geht schluchzend zu einem Begräbnis.

Wie ist es möglich, gewählt zu werden in die Akademie?

La phonologie moderne, depuis l'école linguistique pragoise, a élaboré l'hypothèse du lien étroit entre son et signification. Le langage poétique manifeste cette propriété avec une force particulière. Pour reprendre la formule de B. Pasternak (1965: 18), «ce qui fait la musique du mot, ce n'est pas sa sonorité, mais la relation entre sa sonorité et sa signification». Aussi la traduction poétique, comme la traduction de la prose littéraire à texture phonétique voulue, se propose-t-elle de reconstituer le lien entre la configuration phonique du texte et son sens, et non pas de reproduire les assonances ou l'écriture phonique. On compare l'Ode de Lomonossov de 1747 à l'Ode sur la paix de Ronsard5 pour faire remarquer que dans les deux poèmes on retrouve l'assonance garde-gradov ograda («la clôture des cités»):

Ô paix heureuse

Tu es la garde vigoureuse

Des peuples et de leurs citéz

(Ode sur la paix, 201-203.)

"Царей и царств земных отрада,

Возлюбленная тишина,

Блаженство сел, градов отрада"

/"Ода 1747г.",1-3/.

Or, si chez Ronsard garde est phonologiquement isolé (l'assonance avec vigoureuse des n'est pas évidente), chez Lomonossov ograda est intégré dans le complexe phonique gradov ograda. Il s'ensuit que du point de vue structural, ces deux textes se distinguent plus qu'ils ne se ressemblent.

En traduction, comme en toute création littéraire, la configuration phonique ne prend un sens que si elle accompagne le thème majeur du poème matérialisé dans son mot clé (c'est le cas des «anagrammes» étudiées par F. de Saussure). La traduction dans une autre langue est alors problématique. Le «Rondeau» de Villon peut servir d'illustration :

Janin l'Avenu,

Va t'en aux estuves;

Et toy la venu,

Jenin l'Avenu,

Si te laves nud 
Et te baignes es cuves.

Jenin l'Avenu,

Va t'en aux estuves.

La séquence la venu-l'Avenu-lave nud est appelée par le nom propre autant que par la tonalité badine du thème. Le problème que poserait la traduction dans toute autre langue de ce poème de Villon vient du fait que dans aucune, en vertu de l'arbitraire du signe linguistique, les mots assonant avec le nom propre ne seraient équivalents au sens des mots assonant avec celui dans le texte original.

Le problème se retrouve dans la traduction de vers construits sur l'assonance non pas d'un nom propre (donné, comme dans l'exemple cité, ou obtenu par le déchiffrage d'autres mots du poème, comme c'est le cas des anagrammes) mais d'un nom clé commun. Le poème de Nazim Hikmet (1954: 88) «Mavi gözlü dev» («Le géant aux yeux bleus») reprend le motif folklorique opposant le géant ( $\mathrm{ev}$ en turc), son grand travail (büjük isler: «grand travail») et une petite femme (minna cik kadin) qui quitte le géant pour un nain (oucenin). Le mot clé dev («géant») est repris onze fois dans le poème (plus de 10 pour cent des occurrences des 100 mots du poème). Dev est repris par des rimes telles que devdi-sevdi-evdi, ev-dev, devin-evin, deve-eve, ainsi que par la symétrie de elveda («adieu») et deve dans le vers culminant. La version russe reconstitue le vers libre du poème mais est sans ressources pour récupérer le rôle de dev dans sa structure. C'est qu'ici, comme dans la langue en général, le mot est le point où se croisent signifiant et signifié.

Les virtuoses de la traduction, il est vrai, arrivent parfois à recréer non seulement l'association du signifiant et du signifié mais aussi l'image phonique de l'original. Ainsi, dans la récente version française de «L'Hymne à la peste» de Pouchkine, le vers Les cheminées vers lui tressaillent $^{6}$, dont le sens et la rection verbale surprennent, laisse supposer que tressaillent est apparu, peut-être inconsciemment, en réponse phonique au verbe trechtchat dans le texte russe. Mais ce sont là des cas exceptionnels. Les langues apparentées permettent, il est vrai, de faire valoir la proximité des systèmes phonologiques. C'est le cas, par exemple, des versions polonaises de vers russes par Touvim, ainsi que des versions russes des poèmes de Touvim. Rappelons que Beaudouin de Courtenay avait déjà fait remarquer que les langues apparentées permettent des traductions qui reproduisent non seulement le sens mais aussi l'image phonétique du mot. La différence des systèmes phonologiques (et sémantiques) font, toutefois, que ces traductions laissent à désirer. C'est ce qu'a d'ailleurs montré Roman Jakobson dès son ouvrage sur le vers tchèque comparé au vers russe, qui a fondé la typologie comparée des systèmes métriques.

Sont intraduisibles en principe les aspects phonologiques de la configuration du texte de départ (y compris son mètre) qui tiennent à la structure de la langue de départ et qui se distinguent des traits phonémiques de la langue de la traduction. En peuvent servir d'exemples les cas assez fréquents d'identification par le poète-traducteur du schème rythmique du texte de la langue étrangère avec celui propre à la sienne. La différence dans le rythme entre la version allemande du poème «Les pas» de Paul Valéry, proposée par Rilke, et celle de l'original se laisse expliquer par une interprétation erronée: le dernier vers du poème Et mon cour n'était que vos pas, un vers à huit syllabes pratiquement insensible aux accents des mots, a été pris comme l'équivalent du vers tonique allemand à nombre de syllabes variables: Und mein Herz war nichts als dein Schritt. Inconsciemment, Rilke semble avoir frappé les mots du vers français de l'accent propre au modèle de la prononciation allemande. La discordance fonctionnelle du mètre a entraîné celle des rimes (chez Valéry, elle est fondée sur l'homonymie et la quasihomonymie, ce qui n'est pas le cas des rimes allemandes) et celle du vocabulaire: le 
philosophique Dich-Erwarten de Rilke ne correspond en rien au laconique vous attendre de Valéry; pas plus que l'opposition entre l'infini (unendlich) et le fini (endlich), entre l'être et le néant à douceur d' être et de n'être pas de Valéry:

$\mathrm{Si}$, de tes lèvres avancées,

Tu prépares pour l'apaiser

À l'habitant de mes pensées

La nourriture d'un baiser,

Ne hâte pas cet acte tendre,

Douceur d'être et de n'être pas,

Car j'ai vécu de vous attendre

Et mon cnur n'était que vos pas.

Voici le texte Rilke :

Wenn deine Lippen vielleicht schon vom Weiten

jenem, der in mir sich bergen muss,

seine unendliche Stillung bereiten

endlich in dem nahrenden Kuss,

eile mir nicht zum Vollzuge, dem zarten,

Süsse, drin Sein und Nichtsein stritt,

denn ich lebte vom Dich-Erwarten,

und mein Herz war nichts als dein Schritt

Un exemple contraire d'équivalence métrique est celui des versions par Marina Tsvetaieva des Possédés et de la chanson du président dans «Le banquet pendant la peste» de Pouchkine. Ces traductions, et c'en est la marque, suivent exactement le mètre du texte original (tétramètre choréique et iambique, respectivement), différant de celui des vers français où l'accent, on le sait, signale la fin du groupe de mots, alors qu'en russe il est porté par le mot. Tsvetaieva se conforme aux exigences du vers syllabique français tout en créant, dans sa version de «L'Hymne», un équivalent français au tétramètre iambique russe: lus «à la russe», ses vers français recouvrent entièrement la mesure du vers traduit. Certains vers du texte de Tsvetaieva reproduisent aussi le dessin rythmique des vers de Pouchkine à accents élidés ${ }^{7}$. On peut dire qu'à travers le vers syllabique français, Tsvetaieva percevait inconsciemment le mètre russe, tout comme Rilke, dans la traduction mentionnée, interprétait ce même vers français à travers le vers tonique irrégulier allemand.

La recherche d'un mètre adéquat peut pousser le traducteur à recourir à un rythme inusité dans la poésie indigène. En peuvent servir d'exemples les multiples traductions des chansons de geste françaises en vers libres accentuels ou en vers toniques à longueur variable. C'est le cas, par exemple, de la traduction de «Li coronnement Loois» par Mandelstam. En voici la strophe deux :

Seignor baron, plaireit vos d'une exemple,

D'une chançon bien faite et avenante?

Quant Deus eslist nonante et nuef reiames

Tot le meillor torna en dolce France;

Le mieldre reis ot a nom Charlemagne;

Cil aleva volentiers dolce France;

Deus ne fist terre qui envers lui n'apende;

Il $i$ apent Bavière et Alemaigne,

Et Normandie, et Anjou, et Bretaigne,

Et Lombardie, et Navare, et Toscana. 
"Не хотите ль, господа бароны, извлечь хороший урок

Из прекрасной складной песни, приятной на слух?

Когдь Господь назначил девяносто Девять царств,

Нежнейшее внимание он Франции подарил -

Лучший из государей носит имя - Карл,

Он Францию взял в руки и поднял выше всех.

И все другие земли к его державе льнут

И баварская марка и алеманский круг.

Французские уделы Норман, Анжу,Бретань,

Ломбардское княжество и с Новаггой Тоскань"

Certains de ces vers peuvent être considérés comme des vers syllabo-toniques propres au russe. Ainsi, les vers sept et neuf se laissent interpréter comme constitués de deux demi-vers iambiques à trois pieds avec l'«adjonction» d'une syllabe inaccentuée après la césure ( $\cup-\cup-\cup-\cup / \cup-\cup-\cup-)$. Mais les vers qui assonnent ou riment avec eux sont de purs vers syllabiques de 13 syllabes. Pour reconstruire le mètre ancien français, Mandelstam cherche à rappeler à la vie un mètre délaissé par la poésie russe des deux derniers siècles en faveur du vers syllabo-tonique.

La rencontre de deux traditions métriques apparentées peut aboutir à des résultats semblables à ceux obtenus en linguistique comparée. Ainsi, la reconstruction de la métrique du slave commun dans les récents travaux de Roman Jakobson a suivi la voie tracée par Pouchkine. S'inspirant du cycle de Prosper Mérimée, Pouchkine entreprit de tourner en russe les chants populaires serbo-croates. La comparaison du mètre de ces derniers avec celui de la chanson populaire russe l'amena à chercher un mètre intermédiaire. Comme ces deux traditions conservent le mieux les traits du vers en slave commun, Pouchkine réussit à appréhender les principes de la structure de ce dernier et à les mettre en œuvre dans ses Chants des Slaves occidentaux (Ivanov 1967).

La comparaison synchronique propre à toute démarche traductionnelle permet de mettre en évidence aussi l'évolution diachronique de la poésie. Dans le travail de l'éminent sinologue V. Alexeiev que nous avons mentionné, une interprétation intralinguale diachronique du texte original est proposée comme alternative à «un rapprochement forcé sur un plan isochronique de textes et de langues qui ne se laissent pas rapprocher ${ }^{8}$ \%. Une autre façon de procéder en face d'un texte ancien, c'est de lui trouver une forme archaïque équivalente dans la langue de la traduction. C'est la démarche de Swinburne (1880: 216) traduisant Villon; son texte est même plus archaïque que celui du poète français. Il n'est que de comparer le fragment suivant du «Débat du cuer et du corps» de Villon:

Que penses tu? - Estre homme de valeur. -

Tu as trente ans. - C'est l'aage d'un mullet. -

Est ce enfance? - Nennil. - C'est donc folleur

Qui te saisit? - Par ou? - Par le collet,

Rien ne cognois. - Si fais: mouches en let;

L'ung est blanc, l'autre est noir, c'est la distance.

What art thou, trow? - A man worth praise, perfay. -

This is thy thirtieth year of wayfaring.-

'Tis a mule's age. - Art thou a boy still? - Nay. -

Is it hot lust that spurs thee with its sting.

Grasping thy throat? Know'st thou not anything? -

Yea, black and white, when milk is specked with flies,

I can make out. - 
Tu as trente ans et This is thy thirtieth year of wayfaring montrent assez que la voie choisie par Swinburne conduit à l'échec. C'est que Villon est accessible au lecteur de nos jours non pas par le truchement d'une traduction archaisante, mais d'abord par son rapprochement avec les poètes modernes, tel Apollinaire, dont l'intonation fait écho à celle de Villon. Il n'est que d'écouter Apollinaire:

\section{Avant d'entrer dans ma cellule \\ Il a fallu me mettre nu \\ Et quelle voix sinistre ulule \\ Guillaume qu'es-tu devenu.}

Et c'est ce qui explique les versions modernes de Villon, nullement archaïsantes, celle du célèbre poète Aighi $^{9}$, par exemple. Ce qui permet, en effet, la réussite et la fidélité de la version, c'est non pas la distance chronologique, mais bien la proximité typologique des langages poétiques. L'excellente version du Voyage de Baudelaire par Tsvetaieva ${ }^{10}$ s'explique en partie par la similitude des poétiques des deux poètes qui, chacun, dans le cadre de son époque littéraire, maniaient librement des formes poétiques traditionnelles et n'hésitaient pas à recourir à l'enjambement, non seulement d'un vers à l'autre, mais aussi entre des quatrains, même si cela brisait la syntaxe poétique:

Entendez-vous ces voix, charmantes et funèbres,

Qui chantent: «Par ici! vous qui voulez manger

Le Lotus parfumé! C'est ici qu'on vendange

Les fruits miraculeux dont votre cœur a faim.»

Encore que rappelant les enjambements propres à Tsvetaieva, l'écriture de ces vers rend exactement la structure de la strophe de Baudelaire. Au-delà d'une fidélité littérale, Tsvetaieva retrouve la vivacité du vers baudelairien, le mouvement impétueux des phrases jaillissant l'une de l'autre et s'entrecoupant l'une l'autre, le chevauchement des vers, mots et groupes sonores. La filiation de la poétique de Tsvetaieva et de celle des poètes français issus des «maudits» doit toujours être présente à l'esprit de ceux qui entreprennent la traduction de la poésie française moderne en russe. Ainsi, les enjambements et les images de «La chanson du mal-aimé» annoncent ceux de Tsvetaieva, au point que le lecteur russe des vers comme

Je suis fídèle comme un dogue

Au maître le lierre au tronc

croit y entendre des équivalents français au «Poème de la fin» de Tsvetaieva.

Tout un domaine de faits de langue importants pour la poésie se trouve à la frontière de la sémantique et de la grammaire, la syntaxe notamment. $\mathrm{Au} \mathrm{XX}^{\mathrm{e}}$ siècle, un des principaux courants de la poésie mondiale (représenté en Russie en premier lieu par Khlebnikov) explore les potentialités de la phrase, et tout d'abord des constructions grammaticalement correctes mais constituées de mots inusités dans ces contextes. Or, un des objectifs des recherches linguistiques à partir des années 1920, c'est la découverte d'importantes propriétés de la langue par l'étude d'énoncés tels que Piroten karollieren elatisch, proposé par Carnap, ou de phrases construites selon les règles de la grammaire anglaise mais faites d'unités qui n'entrent pas dans le vocabulaire de la langue, comme chez Carroll (voir Chomsky et Hall 1968: 295).

Bien avant Carnap, l'un des prédécesseurs de la phonologie pragoise, le linguiste russe L. Sčerba, proposait à ses étudiants de l'université de Pétrograd d'analyser des propositions dont les morphèmes grammaticaux étaient portés par des mots et des racines «inventés». 
Ce sont là autant d'exemples qui illustrent l'étonnant parallélisme entre l'évolution des recherches linguistiques, d'une part, et celle du maniement artistique de la langue conduisant à des constructions phrastiques «inventées», analogues à celles en question (au niveau du mot et non pas de la phrase, le même problème, d'ordre phonologique, a été élaboré par Khlebnikov et, après lui, par Auden et d'autres poètes).

Dans les années 1950, les linguistes se sont attaqués à un problème similaire, à savoir l'étude de phrases faites de mots qui, tout en appartenant au vocabulaire de la langue et en étant groupés dans des syntagmes grammaticalement corrects, semblent inusités au plan sémantique. Donnée en exemple par Chomsky dans ses Syntactic Structures, la phrase Green ideas sleep furiously devait susciter toute une littérature, bon nombre de linguistes faisant valoir que la phrase en question pouvait être pensée dans un certain contexte comme un énoncé poétique. D'autres phrases de ce type mentionnées par Chomsky dans son travail suivant ${ }^{11}$ pour illustrer la violation des régularités sémantiques de la langue concernant la temporalité ressemblent fort à des combinaisons du langage poétique, telle all the moon long chez Dylan Thomas.

Dans cette perspective, le problème dont discute Chomsky peut être considéré comme une expérience poétique dont la visée est aussi celle des linguistes: mettre en évidence les rapports entre la syntaxe et la sémantique dans la langue naturelle.

Le premier livre en prose entièrement bâti sur la violation des normes de la conception des situations et de leur évocation verbale a été Alice au pays des merveilles de Carroll. Publiée en 1865, cette œuvre a précédé de peu la rédaction de Maldoror de Lautréamont, amené à la poétique de l'absurde non pas par la réflexion sur les règles de la langue commune et des situations communes par elle évoquées, comme Carroll, mais par la volonté de délivrer le langage poétique de la servitude du langage. La similitude des résultats obtenus par les deux auteurs est vérifiée par le fait que les poètes qui ont prolongé la tradition de Lautréamont ont aussi repris l'expérience de Carroll: l'une des premières traductions de l'œuvre de Carroll fut la version française d'Aragon.

Si les «absurdités» de Carroll ont pour source la réflexion sur la logique des règles linguistiques, dans Alice, elles se manifestent aussi par le quasi-calembour, le jeu de l'homonymie des vocables qui se prononcent autrement qu'ils ne s'écrivent. Les expériences de distorsion sémantique se fondent sur le manque de précision de chaque mot commun isolé, en cela différent du terme scientifique. C'est ce trait propre à la langue qui est largement exploité par le langage poétique, notamment dans la poésie du $\mathrm{XX}^{\mathrm{e}}$ siècle: ses séquences sémantiques se distinguent de celles précédemment acceptées, comme diffère la musique atonale de Schoenberg de la musique classique (cette analogie pourrait être développée à partir des derniers acquis de la sémantique linguistique). Pour la poésie européenne moderne, les particularités sémantiques mentionnées prennent une importance d'autant plus grande que la rime, le rythme et les autres facteurs phoniques ne jouent plus le rôle qui était le leur dans le passé. C'est dire que celui qui traduit bon nombre de poètes modernes doit s'attacher d'abord à une modulation sémantique des mots dans la langue d'arrivée qui permette d'atteindre l'équivalence avec le texte original. Que cet effort impose un supplément de création est évident, mais le produit d'une telle traduction expérimentale serait un apport essentiel à l'enrichissement du langage poétique.

Notes

1. Voir A. Dorogov, V. Ivanov, B. Ouspenski : «P. Florenski et son article "La perspective inverse"», travaux de 1'Université de Tartu, vol. 198, Travaux sémiotiques, III, Tartu, 1967 (en russe).

2. V. Alexeiev: «Une nouvelle méthode et un style nouveau de traductions en langues européennes des classiques chinois anciens», Les travaux de l'académicien V. M. Alexeiev. Compte rendu des recherches en 
46. Section de littérature et de langue de l'Académie des sciences de l'URSS, Moscou-Léningrad, 1947, p. 29; idem, La littérature chinoise, Moscou, 1978, p. 423 et passim (en russe).

3. La somme des idées apportées par A. Kolmogorov à la théorie de l'information a été exposée dans son article «Trois approches de la définition de la notion "quantité d'information"»; Problemy peredaci informacii (Problèmes du transfert d' information), t. I, 1965, fasc. I (en russe).

4. César Vallejo 1958, Poemas scogidos, Editura Latinoamericana, p. 80 . Version de H. M. Enzenberger (1964), Museum der modernen Poesie, Deutscher Taschenbuch Verlag, p. 328.

5. L. Poumpiansi (1935), «Lomonossov et Malherbe», xVII vek (Le XVIII siècle), I, p. 128, note, V. Ivanov (1961), «Problèmes linguistiques de la traduction poétique» - «La traduction mécanique», Travaux de l'Institut de mécanique de précision et d'équipement informatique de l'Académie des sciences de l'URSS, fasc. 2, Moscou, p. 382 (en russe).

6. La poésie russe, édition bilingue, Paris, 1965, p. 102. Pour l'analyse détaillée de la complémentarité (selon Niels Bohr) des versions de ce poème, voir V. Ivanov, «Notes sur les traductions par Marina Tsvetaieva de l'Hymne en l'honneur de la peste et des Possédés de Pouchkine», Masterstvo perevoda 1966 (Maîtrise de la traduction), Moscou, 1968, p. 389-402; idem, «Les causes langagières des difficultés de la traduction littéraire», Problèmes actuels de la théorie de la traduction littéraire, t. 2, Moscou, 1967, p. 265-269 (en russe).

7. Sur cette particularité du rythme de Tsvetaieva, voir V. Ivanov, «Le mètre et le rythme», dans Le poème de la fin de T'svetaieva, Teorija stikha (La Théorie du vers), Léningrad, 1968 (en russe).

8. V. Alexeiev, Une méthode et un style nouveau de traduction en langues européennes des classiques chinois anciens, p. 29 (en russe).

9. Poètes français des $\mathrm{XV}$-XX' siècles. Traductions et notices biographiques par Gennadij Aigui, Tcheboksary, 1968, p. 8-11.

10. Pour les détails, voir V. Ivanov. Sur les versions de Marina Tsvetaieva, préface au livre de Marina Tsvetaieva, Prosto serdce (Le Cour, simplement), Moscou, 1967 (en russe).

11. N. Chomsky (1965), Aspects of the Theory of Syntax, Cambridge, Mass., p. 77. Chomsky n'a pas remarqué que ses exemples d'incongruité purement sémantique se réfèrent à des relations de temps.

\section{BIBLIOGRAPHIE}

ALEXEIEV, V. (1916): Un poème chinois sur le poète, Petrograd; Un poète-paysagiste chinois évoque son inspiration et son paysage. Zvezda, no 12, p. 143-150 (en russe).

CHAO, Yuen-Ren (1958): «The non-uniqueness of phonemic solutions of phonetic systems», Readings in Linguistics, (Joos éd.), 2e éd., New York.

CHOMSKY, N. et M. HALLE (1968): The Sound Pattern of English, New York.

FLORENSKI, P. (1922): Les Nombres imaginaires en géométrie, Moscou (en russe).

HIKMET, Nazim (1954): Seçilmissürler, Sofia.

IVANOV, V. (1967): «Notes sur la poétique comparative et historique indo-européenne», For Roman Jakobson, Paris, La Haye.

PASTERNAK, B. (1965): «Notes d'un traducteur», Ruskaja literatura (La Russie littéraire), no 13 (117) (en russe),

SWINBURNE, A. C. (1880) : Poems and Ballads, second series, 3e éd., Londres. 